\title{
ALERIDAD Wikipedia en las facultades de educación españolas. La visión de los estudiantes universitarios
}

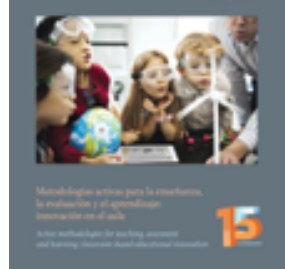

\author{
Wikipedia at the spanish faculties of education. \\ The vision of university students
}

D Dr. Ángel Obregón-Sierra es docente e investigador de la Universidad Isabel I (España) (angel.obregon@uil .es) (https://orcid.org/0000-000 1-8801-317X)

(1) Dra. Natalia González-Fernández es docente e investigadora de la Universidad de Cantabria (España) (gonzalen@unican.es) (https://orcid.org/0000-0001-6669-8446)

Recibido: 2019-09-05 / Revisado: 2020-06-02 / Aceptado: 2020-06-05 / Publicado: 2020-07-01

\section{Resumen}

Desde los comienzos del siglo $X X \mid$ se ha constatado un cambio en la forma que tienen las personas de conectarse a Internet, que interactúan más con los creadores de las páginas web y dedican más horas a conectarse a varias herramientas que se han denominado Web 2.0, como las redes sociales, los wikis y los blogs. Uno de los wikis más conocidos es la Wikipedia, enciclopedia en línea libre editada colaborativamente por usuarios de todo el mundo. En esta investigación hemos aplicado un cuestionario en línea diseñado ad hoc para constatar si los estudiantes universitarios españoles usan esta herramienta, si la conocen y si la conceden importancia de cara a sus estudios. Los resultados han sido obtenidos tras la cumplimentación de |173 cuestionarios por parte de estudiantes universitarios de Educación en toda España. Los alumnos afirman usar mucho las redes sociales, los documentos en línea y la Wikipedia. Solo un 5,4\% de los encuestados indicó que no utiliza esta herramienta. Aseguran conocer su funcionamiento, y tan solo un 1,74\% admitió desconocerla totalmente. Sin embargo, no la dan mucha importancia para sus estudios, como al resto de herramientas de la Web 2.0. Además, la mayoría sabe que pueden editar en ella, pero no se ven capaces de aportar información, por lo que el número de editores es muy bajo.

Descriptores: Wikipedia, wikis, educación superior, estudiantes universitarios, trabajo colaborativo, cuantitativo.

\section{Abstract}

Since the beginning of the 21 st century, there has been a change in the way people connect to the Internet, interacting more with the creators of web sites and spending more time connecting to several tools that have been called Web 2.0, such as social networks, wikis and blogs. One of the best-known wikis is Wikipedia, free online encyclopedia collaboratively edited by users around the world. In this research we have applied an online questionnaire designed ad hoc to see if Spanish university students use this tool, if they know it and if they attach importance to their studies. The results have been obtained after the completion of I|73 questionnaires by university students of Education throughout Spain. Students say they use social networks, online documents and Wikipedia. Only $5.4 \%$ of respondents indicated that they do not use this tool. They make sure they know how it works, and only 1.74\% admitted to not knowing it completely. However, they do not give it much importance to their studies, as the other Web 2.0 tools. In addition, most of them know that they can edit it, but they cannot provide information, so the number of editors is very low.

Keywords: Wikipedia, wikis, higher education, university students, collaborative writing, quantitative.

Forma sugerida de citar: Obregón-Sierra, Á., \& González-Fernández, N. (2020). Wikipedia en las facultades de educación españolas. La visión de los estudiantes universitarios. Alteridad, 15(2), 218-228. https://doi.org/10.17163/alt.v15n2.2020.06 


\section{Introducción}

Una de las herramientas más importantes de Internet es la Wikipedia, que habitualmente se encuentra entre las diez páginas más consultadas del mundo (Alexa, 2020), y lo es también entre los estudiantes y profesores universitarios (Brox, 2019; Wannemacher \& Schulenburg, 2010). Tras introducir 26 términos aleatorios de diferentes áreas del conocimiento en el buscador de Google constatamos que tan solo uno de estos no mostraba en la primera posición el artículo de la Wikipedia referente a dicho término, demostrando la facilidad de acceso que esta enciclopedia virtual ofrece a los usuarios de Internet.

El número de personas que acceden a consultar información en Internet en temas tan relevantes como la salud y la educación ha aumentado considerablemente en tan solo cuatro años, según las encuestas realizadas entre 2015 y 2019 por el Instituto Nacional de Estadística (Instituto Nacional de Estadística, 2019). Otra encuesta, realizada entre estudiantes universitarios de Estados Unidos, mostró que más de la mitad consultaban frecuentemente la Wikipedia, incluyendo su utilización en trabajos de investigación, aunque principalmente en los momentos iniciales de estos (Head \& Eisenberg, 2010).

En Francia, los usuarios más confiados a la hora de consultar Wikipedia son los estudiantes, mientras que los menos confiados son los trabajadores encuadrados en profesiones intelectuales superiores. A su vez, los jóvenes entre 18 y 24 años eran los más confiados en comparación con el resto de rangos de edad (Foglia, 2008). Ambos casos son similares a los expuestos en investigaciones desarrolladas en otras universidades, que constatan un uso de la Wikipedia generalizado en los estudiantes (Margaryan et al., 2011; Pozzali \& Ferri, 2010; Shen et al., 2013).

En España los datos son similares. En la Universidad de Oviedo la Wikipedia es consultada como primera opción por un $15 \%$ de los alumnos, como segunda opción por un 35\% y como tercera opción por un 25\% (Alonso \&
García, 2013), mientras que en la Universidad de Salamanca más del 75\% la utiliza ocasionalmente, y el 53\% afirma hacerlo en sus trabajos de clase (Sánchez, 2014).

Son numerosos los autores y profesores contrarios a la utilización de la Wikipedia en las aulas, y en general para la búsqueda de información, influenciando negativamente a sus alumnos (Garrison, 2018). Varios centros educativos han declarado tener problemas con algunos contenidos de la Wikipedia y la confusión de muchos profesores respecto a su utilización también debe ser tenida en cuenta (Meishar-Tal, 2015).

Muchos estudiantes en su último curso de secundaria (18-19 años) admiten que sus profesores no permiten utilizarla, pero aun corriendo el riesgo de encontrar datos erróneos prefieren utilizarla porque posee el conocimiento que ellos necesitan (Valverde-Crespo et al., 2019). Los estudiantes de secundaria, en ocasiones, presentan trabajos copiados literalmente o con muy pocas modificaciones, además de no mostrar en ellos las referencias de donde obtuvieron dicha información (Sormunen \& Lehtiö, 2011).

Otros motivos aducidos para no utilizarla son la falta de garantía de fiabilidad, que es una fuente no cualificada, abundantes contenidos incompletos o incoherentes, genera pereza intelectual, favorece el copia-pega, posee artículos esquemáticos y la baja calidad narrativa (García, 2010). El profesor Pierre Assouline indicaba en el prefacio del libro La Révolution Wikipédia (Gourdain et al., 2007) que los estudiantes no saben cómo se realizaba una investigación documental antes de existir la Wikipedia. Sin embargo, esta no habría de sustituir a la investigación documental. La Wikipedia no es una fuente primaria, por lo que cualquier usuario debe consultar las fuentes primarias que indican los artículos en su parte inferior (Los cinco pilares, 2020).

Las dudas acerca de su calidad son uno de los mayores impedimentos para los profesores (Chen, 2010), aunque también se encuentran otras causas como son la imagen social de la Wikipedia, la poca aceptación en las instituciones 
educativas y el poco apoyo que se recibe para la formación en el empleo de aplicaciones como la Wikipedia. También es importante tener en cuenta la notable influencia que puede ocasionar la opinión de un individuo en su entorno, ya que la opinión de los profesores influye en la de sus estudiantes (Lim, 2013), e incluso otros docentes suelen influir en la opinión de sus compañeros, porque una opinión positiva podría generar que los compañeros también la usen (Eijkman, 2010; Lladós et al., 2013). Algún autor ha llegado a afirmar que un grupo de personas que trabajan de forma gratuita dificulta las perspectivas de trabajo para los estudiosos, y que por este motivo son más críticos con la Wikipedia (Hastings-Ruiz, 2015).

Constatamos que a medida que transcurre el tiempo, la cantidad de investigaciones son más numerosas (Park, 2011), las actividades con la Wikipedia en el aula son más habituales (Brailas et al., 2015; Brox, 2016; Christensen, 2015; Dawe \& Robinson, 2017; Di Lauro \& Johinke, 2016; Petrucco, 2019; Zou et al., 2020) y las opiniones de los expertos se tornan más positivas que los primeros estudios (Barnhisel \& Rapchak, 2014; Josefsson et al., 2014; Soler-Adillon et al., 2018). En los últimos estudios incluso se afirma que el uso académico activo de la Wikipedia tiene una influencia positiva en el rendimiento académico de los estudiantes (Meseguer-Artola et al., 2019), recomendando en todo caso prudencia a la hora de consultar las fuentes (Eijkman, 2010).

Los alumnos universitarios admiten que la Wikipedia es fiable y útil, aunque lo que más valoran es su actualidad, en contraposición con la completitud (Meseguer-Artola, 2014). En Australia, el 24\% de los estudiantes universitarios la considera muy útil, y el $87,5 \%$ la utilizan para los trabajos de clase (Selwyn \& Gorard, 2016). Una de las investigaciones más elaboradas en cuanto al uso de la Wikipedia por los estudiantes universitarios en Estados Unidos fue el trabajo realizado por Head y Eisenberg (2010), que constataron que el $30 \%$ la consultaban siempre, el $22 \%$ frecuentemente y el $23 \%$ ocasionalmente.

Respecto al porcentaje de editores que hay entre los estudiantes, estos se encuentran en un nivel muy bajo, el $1 \%$ admite editar frecuentemente, un $20 \%$ lo hace rara vez, mientras que el $77 \%$ no lo ha hecho nunca. El 0,3\% crea artículos frecuentemente, mientras que el $83 \%$ nunca lo hizo (Kleimann et al., 2008). Los alumnos de estudios de agricultura fueron los que mayor porcentaje de fiabilidad otorgaron a la Wikipedia, un $69 \%$, mientras que los resultados entre estudiantes de filosofía la otorgaron un $38 \%$ de fiabilidad.

La realidad es que solo una quinta parte de los estudiantes desde secundaria hasta la universidad ha recibido explicaciones del funcionamiento de la Wikipedia, y de estos, más de un 30\% fueron asistidos por amigos o familiares (Sahut et al., 2015), lo que puede ocasionar problemas si dichas personas no disponen de conocimientos profundos en la temática. En la misma línea encontramos las investigaciones de Forte y Bruckman (2010), que afirman que los estudiantes no han sido formados debidamente, y por ese motivo ven una complejidad añadida al hecho de aportar citas a sus trabajos. El alumnado consulta fuentes que no consideran fiables, e incluso no saben utilizarlas (Judd \& Kennedy, 2011), mientras que la reacción de los profesores es más pasiva que activa en el proceso de enseñarles a utilizarla (Meishar-Tal, 2015). Los estudiantes, en multitud de ocasiones van a consultar la Wikipedia aun admitiendo que no es fiable, pero al estar bien posicionada y poseer casi cualquier artículo los anima a seguir utilizándola (Valverde-Crespo \& González-Sánchez, 2016).

Atendiendo a esta realidad, presentamos este trabajo, donde queremos determinar qué percepción tiene el alumnado universitario sobre el conocimiento, uso e importancia que le otorga a la Wikipedia en su formación académica.

\section{Metodología}

\subsection{Instrumento y procedimiento}

Diseñamos y validamos una herramienta de recogida de información cuantitativa, un cuestionario ad hoc (Obregón \& González, 2016). Se realizaron estudios de validez de contenido mediante una 
prueba piloto, cumplimentada por 73 estudiantes de primer curso del Grado de Magisterio en las especialidades de Educación Primaria e Infantil, $y$ un panel de expertos, tal y como se propone en la técnica Delphi, compuesto por diez investigadores universitarios. En el cálculo de la fiabilidad se obtuvo un resultado de ,878 en el Alpha de Cronbach. El cuestionario estaba compuesto por 93 ítems al comienzo del diseño y validación, y 79 en la versión final, tras tener en cuenta las propuestas sugeridas por los jueces expertos, atendiendo a criterios de claridad, pertinencia, orden y eficacia. Esta última versión se distribuyó en cuatro dimensiones: Datos personales, parte general de Internet y la Web 2.0, parte específica de la consulta y la edición de la Wikipedia. Fue enviado a través de correo electrónico, previo consentimiento de los decanos de las facultades de Educación implicadas, mediante el enlace a la aplicación en línea del mismo.

Las informaciones cuantitativas proporcionadas por los ítems del cuestionario fueron tratadas con el programa SPSS 19 para los análisis de fiabilidad, estadística descriptiva, inferencial (pruebas de Kruskal-Wallis, Wilcoxon y U de Mann-Whitney) y relacional (coeficientes Tau de Kendall y Rho de Spearman), explorando el uso que los estudiantes hacen de la Wikipedia, el conocimiento que tienen de ella y la importancia que la otorgan para sus estudios.

\subsection{Participantes}

El número de estudiantes en los estudios de grado, primer y segundo ciclo matriculados en todas las universidades públicas y privadas de España en el área de Educación fue de 156.986 estudiantes. Con estos datos, el número mínimo de cuestionarios a cumplimentar era de 1060, obteniéndose 1415 respuestas una vez terminado el plazo de entrega. Tras eliminar las respuestas invalidadas, esta investigación analiza los datos aportados por 1173 estudiantes universitarios de Educación en España.

Tal y como puede observarse en el gráfico 1, participaron 959 mujeres y 214 hombres, siendo en su mayoría (66\%) menores de 23 años. Estuvieron representados todos los cursos de la formación universitaria, en las titulaciones de grado en Magisterio en Educación Primaria $(36,4 \%)$, Infantil (23,4\%), Pedagogía (13,2\%), Educación Social $(11,2 \%)$, Infantil y Primaria $(3,8 \%)$ y también en varios tipos de máster $(11,9 \%)$. Contestaron al cuestionario más de la mitad de las universidades españolas, destacando las respuestas de las universidades del este peninsular, ya que entre las universidades de la Comunidad Valenciana y Cataluña sumaron el $40 \%$ del total.

\section{Gráfico 1. Estudiantes universitarios que participaron en el cuestionario}

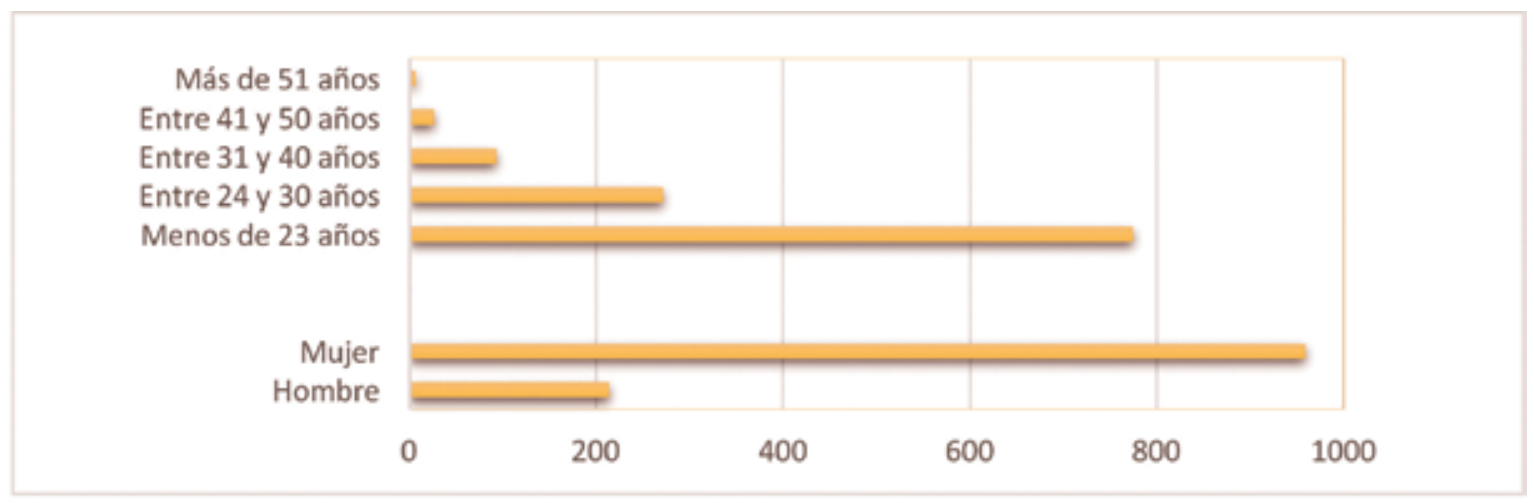

Fuente: Elaboración propia (2020) 


\section{Resultados}

El cuestionario se utilizó como herramienta de recogida de información para conocer la realidad actual acerca del uso, importancia y conocimiento de las herramientas de la Web 2.0, y específicamente de la Wikipedia, por parte de los estudiantes universitarios españoles en estudios de Educación. Se preguntó a los estudiantes por las redes sociales, redes sociales basadas en vídeos, microblogging, blogs, wikis, Wikipedia, plataformas de almacenamiento, documentos en línea y foros.

A partir de las respuestas obtenidas hemos observado que los alumnos universitarios en España utilizan muy frecuentemente las redes sociales como Facebook o Instagram, en concreto un $96,3 \%$ admitió usarlo poco, bastante o mucho. Las redes sociales basadas en vídeos como YouTube, los documentos en línea y la Wikipedia, también son muy usados, pero no así los blogs, wikis y foros. Esto puede ser debido a que los foros y los blogs han sido sustituidos por las redes sociales, en las que es posible opinar, comentar y seguir a otras personas de una manera más sencilla.

Tras realizar las pruebas de Kruskal-Wallis y U de Mann-Whitney aplicadas a la dimensión "Uso_Redes_Sociales", observamos que los alumnos más jóvenes y en cursos más bajos utilizan más las redes sociales $(\mathrm{p}<, 003$ entre los menores de 23 años y el resto de grupos de edad y $\mathrm{p}<, 008$ entre el grupo de edad de 24 a 30 años y los más mayores). A medida que aumentan de curso ( $\mathrm{p}=$ ,012 entre los estudiantes de primero y de cuarto y $\mathrm{p}=, 040$ entre los estudiantes de segundo y cuarto) o adquieren una titulación dejan de utilizarlas con tanta frecuencia ( $\mathrm{p}<, 036$ en todos los casos comparando los estudiantes sin titulación y los que tienen C.F.G.S., diplomatura o licenciatura). Cuanto más tiempo navegan los estudiantes universitarios en Internet ( $p=, 000$ en todos los casos en los que se comparan las horas dedicadas) y a medida que aumenta su conocimiento informático $(\mathrm{p}=, 002$ entre el nivel básico y el avanzado y $\mathrm{p}=, 005$ entre el nivel medio y el avanzado), más utilizan las herramientas de la Web 2.0. En cuanto a las herramientas para la transferencia de archivos, los documentos en línea y los foros, los estudiantes menores de 23 años son los que menos utilizan este tipo de herramientas ( $\mathrm{p}=$ ,000 entre los menores de 23 años y entre los que tienen una edad entre 24 y 30 años y $p=, 001$ entre los menores de 23 años y los que tienen entre 31 y 40 años), y su uso aumenta a medida que aumenta la edad. El alumnado que cursa estudios de máster, y los que ya tienen una titulación superior, también utilizan con más frecuencia este tipo de herramientas comparándolo con los estudiantes que solo disponen del título de bachiller $(\mathrm{p}<, 008$ entre los estudiantes sin titulación y los licenciados o posgraduados, $\mathrm{p}=, 006$ entre los estudiantes con C.F.G.S. y los que tienen título de posgrado).

La utilización de la Wikipedia es intermedia, siendo de 2,61 su media, ligeramente superior a la de la escala $(2,5)$. Los estudiantes que menos usan la Wikipedia son los que ya disponen de un ciclo formativo, en comparación con los estudiantes que poseen otro tipo de titulación (bachiller, licenciatura y posgrado). Constatamos además que los estudiantes con nivel informático básico consultan menos la Wikipedia (media de $2,49)$, y según va aumentando su nivel de conocimiento aumenta su uso (3,11 los expertos). Por último, los usuarios que dedican más de cuatro horas a conectarse a Internet usan más la Wikipedia que el resto de usuarios que navegan menos tiempo ( $p<, 025$ en todos los casos comparando con el resto de opciones). Además, se encontraron diferencias entre los estudiantes con nivel básico de conocimiento informático con respecto a los que afirmaban tener un nivel avanzado $(\mathrm{p}=, 012)$ y experto $(\mathrm{p}=, 004)$, los de nivel medio con los expertos $(\mathrm{p}=, 008)$ y los avanzados con respecto a los expertos $(\mathrm{p}=, 034)$. 
Gráfico 2. Uso que le dan los estudiantes universitarios a nueve herramientas

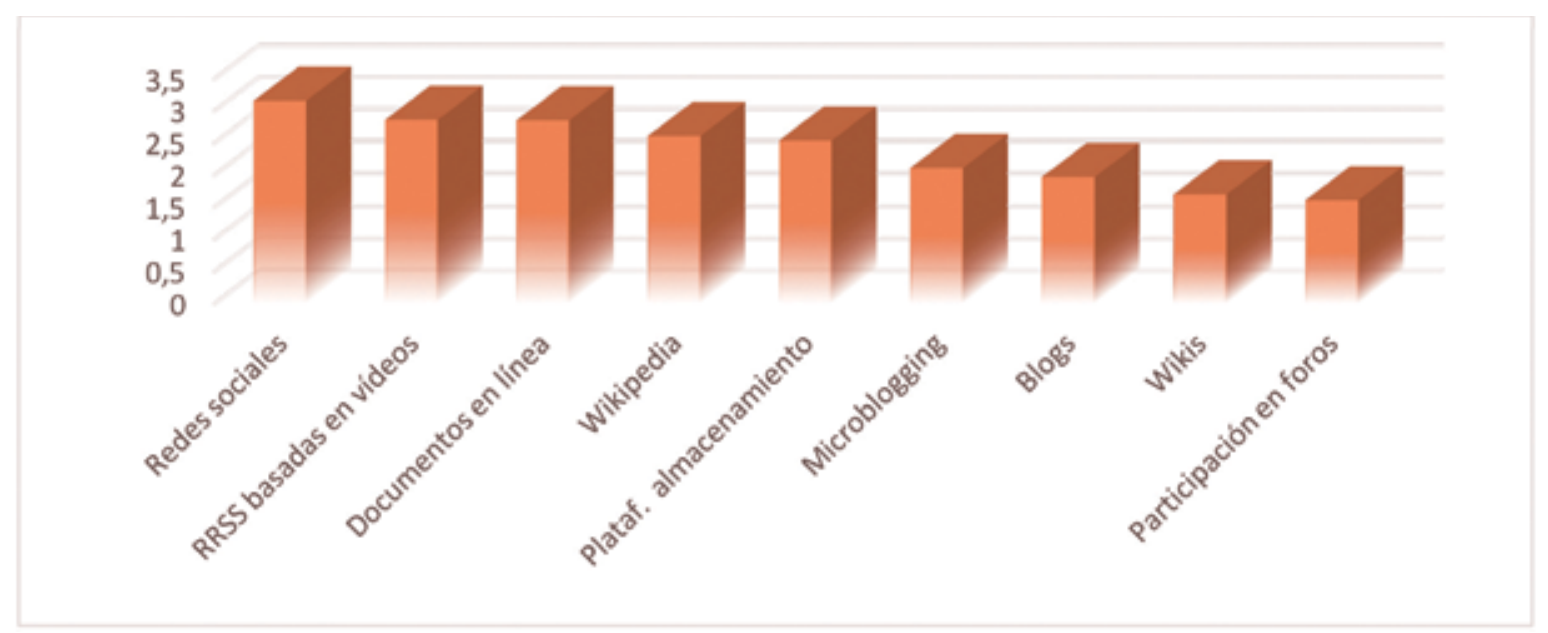

Fuente: Elaboración propia (2020)

El conocimiento de las herramientas Web 2.0 por parte de los estudiantes universitarios en España es medio, debido en parte al desconocimiento en dos de sus principales herramientas: Los foros y los wikis. El conocimiento en el resto de herramientas es medio, exceptuando el caso de las redes sociales como Facebook o Instagram, en las cuales admiten poseer conocimientos altos. Hemos observado también que el conocimiento de los estudiantes en las herramientas de la Web 2.0 es mayor cuanto más tiempo dedican a conectarse a Internet ( $\mathrm{p}=, 000$ en todos los casos de comparación entre las horas dedicadas) y mayor conocimiento informático poseen $(\mathrm{p}=, 000$ en todos los casos comparados de los niveles informáticos). Además, los más jóvenes afirman tener un mayor conocimiento de las herramientas Web 2.0 respecto a los estudiantes mayores $(\mathrm{p}=, 010$ entre los menores de 23 años y los de 31-40 años, $\mathrm{p}=, 012$ entre los menores de 23 años y los de 41-50 años, $\mathrm{p}=, 034$ entre los estudiantes de 24-30 y los de 31-40, y $\mathrm{p}=, 024$ entre los estudiantes de 24-30 y los de 41-50 años), mientras que a medida que superan los cursos de grado su conocimiento aumenta $(\mathrm{p}=, 008$ entre primero y segundo y $\mathrm{p}=$ ,014 entre primero y cuarto curso).

En cuanto al conocimiento que tienen los estudiantes universitarios españoles de la
Wikipedia, podemos afirmar que es medio-alto, tal y como indica la media de este ítem $(2,88)$ y el hecho de que solo el 1,74\% afirma tener un conocimiento nulo de la Wikipedia. Se ha constatado que cuanto mayor es el nivel informático que poseen los estudiantes, más conocimiento tienen sobre la Wikipedia, llegando hasta un conocimiento muy alto de los expertos en informática ( $\mathrm{p}=, 000$ en todos los casos comparados excepto $\mathrm{p}=, 006$ entre los avanzados y los expertos). Además, observamos el mismo efecto en los estudiantes que dedican más horas a navegar por Internet, cuantas más horas empleadas, mayor conocimiento afirman poseer $(\mathrm{p}<, 020$ en las comparaciones entre estudiantes que dedican menos de una hora y el resto, $\mathrm{p}=, 002$ entre los que dedican 1-2 horas y los que dedican más de 4 horas y $\mathrm{p}=, 010$ entre los que dedican entre 2 y 4 horas y los que dedican más de 4 horas). En cuanto a la correlación entre el uso de la Wikipedia y el conocimiento que tienen de ella existe una correlación moderada $(\rho=, 479$ fue el resultado del coeficiente de correlación Rho de Spearman). 


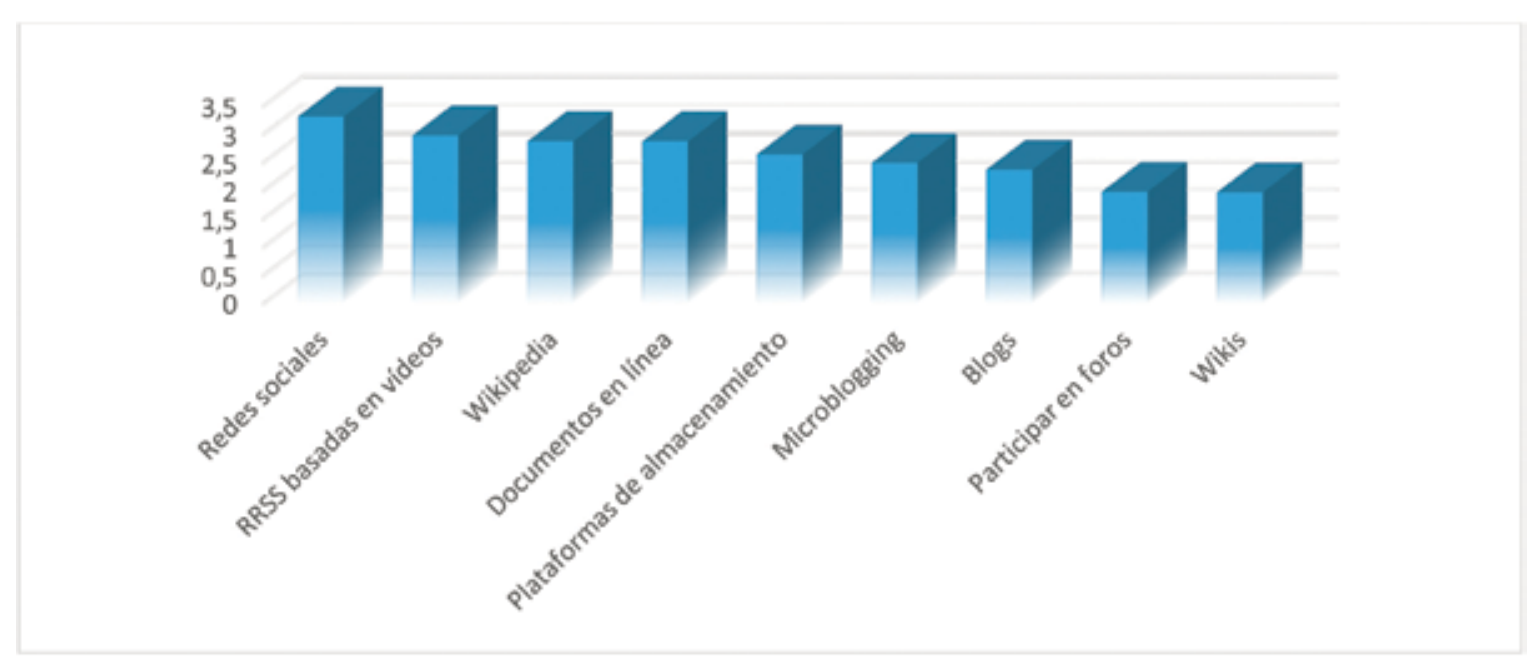

\section{Fuente: Elaboración propia (2020)}

Los estudiantes universitarios españoles otorgan poca importancia para sus estudios a los foros, las redes sociales o los blogs. Sin embargo, la navegación por Internet es muy importante para ellos. Si tenemos en cuenta que esta no es una herramienta de la Web 2.0 concluimos que el alumnado otorga poca importancia para sus estudios a este tipo de herramientas (la media, sin incluir la navegación por Internet es de 2,10). Ciertos estudiantes consideran a las herramientas Web 2.0 más importantes que otros, como es el caso de los mayores de 23 años ( $\mathrm{p}<, 033$ comparando con el resto de grupos de edad), los alumnos que estudian en las universidades del sur del país ( $\mathrm{p}<, 035$ comparando con el resto de universidades del país), los que cursan estudios de máster $(\mathrm{p}<, 037$ comparando con el resto de especialidades), los que dedican más horas a navegar ( $p<, 016$ en todas las comparaciones realizadas exceptuando entre los alumnos que se conectan entre 1-2 horas y entre 2-4 horas que devolvió un $\mathrm{p}=, 215)$, los que tienen mayores conocimientos en informática ( $p<, 008$ en todos los casos exceptuando entre los que tienen nivel avanzado y experto que fue de $\mathrm{p}=, 057$ ) y los que ya poseen una licenciatura $(\mathrm{p}<, 033$ en todos los casos comparados).
El resultado del análisis de la importancia que otorgan los universitarios de Educación a la Wikipedia en sus estudios nos permite afirmar que los estudiantes universitarios españoles otorgan una importancia intermedia-baja a la Wikipedia. La media es de 2,37, los valores de la mediana normalmente son de 2,00 y solo en seis casos los valores de las categorías de cada ítem superan la media de la escala $(2,5)$. Los hombres $(\mathrm{p}=, 010)$, los alumnos entre 31 y 40 años en comparación con los menores de 31 ( $p=, 002$ en ambos casos), los que estudian en una universidad del sur de España $(\mathrm{p}<, 043$ en todos los casos), los que disponen de un título de licenciatura, comparado con los que tienen el título de bachillerato $(\mathrm{p}=, 002)$ o un C.F.G.S $(p=, 006)$, los que tienen un nivel de conocimiento informático de experto con respecto a los de nivel intermedio $(\mathrm{p}=, 032)$ y básico $(\mathrm{p}=$ ,014), y los que dedican más de 4 horas a navegar por Internet, comparado con los que dedican entre 1-2 horas $(\mathrm{p}=, 022)$ y entre $2-4$ horas $(\mathrm{p}=, 032)$ dan más importancia a la Wikipedia para sus estudios que el resto de categorías.

Además de estos datos, el 92,2\% de los estudiantes universitarios sabía que cualquier persona puede editar en la Wikipedia, pero un 
5\% no lo sabían y la utilizan bastante o mucho. Respecto a la cantidad de estudiantes que han editado en alguna ocasión en la Wikipedia, solo han sido el $12 \%$ del total, mientras que un $0,38 \%$ lo ha hecho frecuentemente o muy a menudo.

Gráfico 4. Importancia que le otorgan los estudiantes universitarios a seis herramientas

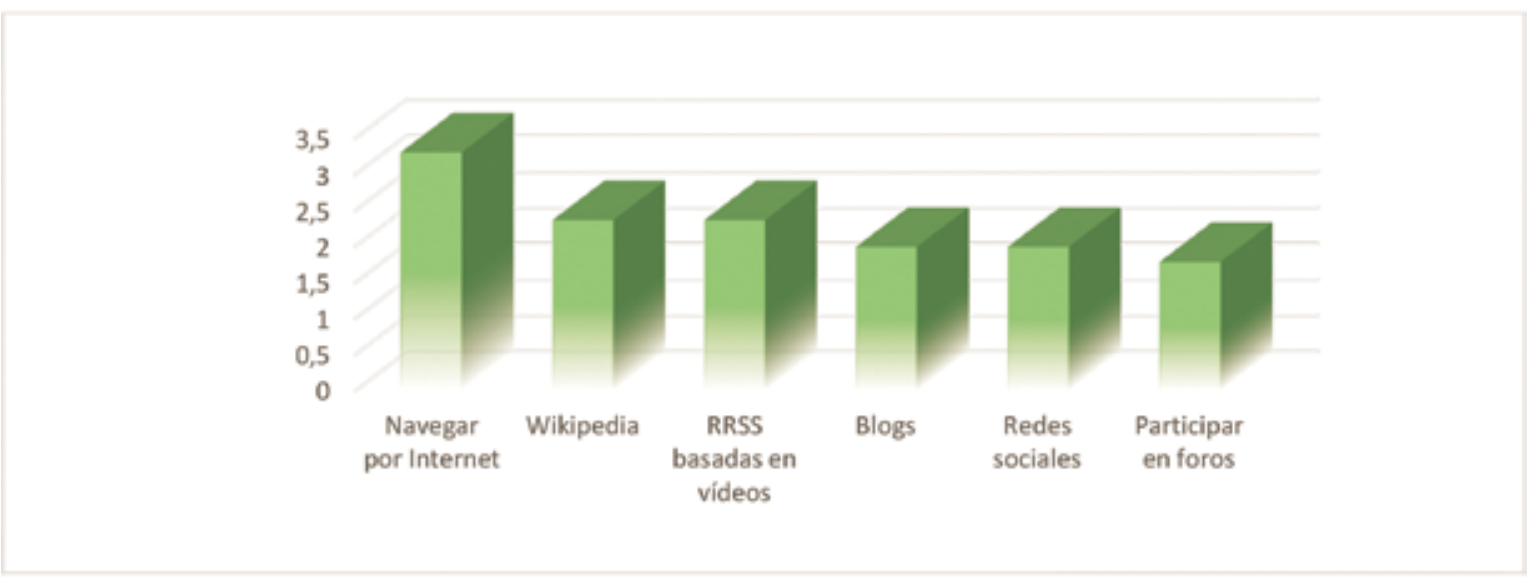

Fuente: Elaboración propia (2020)

\section{Discusión y conclusiones}

El objetivo de esta investigación se centraba en conocer la realidad actual acerca del uso, importancia y conocimiento de las herramientas de la Web 2.0, y específicamente de la Wikipedia, por parte del alumnado que cursa estudios en las Facultades de Educación españolas. A partir de los resultados del cuestionario, es posible comprobar que un porcentaje alto de estudiantes utilizan las redes sociales $(96,3 \%)$, dato similar a los aportados por el Instituto Nacional de Estadística (2019), el cual indica un $91,1 \%$. También es destacable el alto número de estudiantes que utiliza las redes sociales, los documentos en línea y la Wikipedia, en contra del bajo uso que dan a los blogs, wikis y foros.

Varias investigaciones han apuntado que más del $80 \%$ de los estudiantes utiliza la Wikipedia (Gilles, 2015; Sánchez, 2014). La media obtenida por una investigación realizada en la Universidad de Salamanca fue de 2,81 (Sánchez, 2014), mientras en nuestro caso ha sido de 2,61, ligeramente inferior, pero por encima de la media de la escala. Existe una mayor coincidencia con la investigación de Gilles (2015), en la cual se afirma que el 5,7\% de sus encuestados no utilizaba la Wikipedia, por el $5,4 \%$ que hemos obtenido en nuestro caso, lo que demuestra un uso habitual de ella.

Respecto al conocimiento e importancia de la Web 2.0 y la Wikipedia observamos que los estudiantes universitarios españoles poseen un conocimiento medio en la mayoría de las herramientas de la Web 2.0, exceptuando en las redes sociales, de las que afirman tener un conocimiento alto. Respecto a la Wikipedia, su conocimiento es medio-alto y solo el 1,74\% afirma tener un conocimiento nulo sobre ella. El uso que hacen de la enciclopedia les confiere, según ellos, conocimiento de la herramienta y de su funcionamiento interno.

Apenas otorgan importancia para sus estudios a las herramientas de la Web 2.0, destacando la baja importancia que conceden a los foros, las redes sociales y los blogs. Principalmente, para sus estudios utilizan otros recursos como los libros de clase, las preguntas al profesor y a sus compañeros, o la navegación por Internet, al 
igual que ya apuntó Gilles (2015). La Wikipedia principalmente es utilizada para temas de ocio, pero le confieren una importancia intermediabaja para sus estudios.

Flanagin y Metzger (2011) constataron que, aunque la mayoría de los estudiantes encuestados conocía la Wikipedia, un $22 \%$ no sabía que todo el mundo podía participar. Este porcentaje ha descendido debido al mayor conocimiento que poseen los estudiantes universitarios del funcionamiento de la Wikipedia, ya que en esta investigación se ha encontrado que tan solo un 7,8\% no sabían que podían editar.

En cuanto al porcentaje de participación, Kleimann et al. (2008) obtuvieron que tan solo un $0,3 \%$ de los estudiantes creaban artículos frecuentemente, mientras que el $83 \%$ no lo habían hecho nunca. Cuquet y García-San Pedro (2019) afirmaron que un 7\% de los estudiantes de ESO, y un $14 \%$ de Bachillerato, habían editado en alguna ocasión, mientras en nuestro caso, solo el $12 \%$ lo había hecho en alguna ocasión, siendo tan solo un $0,38 \%$ que lo ha hecho frecuentemente o muy a menudo. El principal motivo que exponen para no hacerlo es que no creen que puedan aportar nada.

En definitiva, este artículo contribuye a una mejor comprensión sobre el conocimiento que tienen los estudiantes de las herramientas tecnológicas que utilizan diariamente. La Wikipedia ofrece una gran diversidad de posibilidades, posibilitando el aprendizaje colaborativo, $y$ fomentando un aprendizaje activo.

Como limitaciones de este estudio podemos indicar el hecho de que la recolección de datos se centró únicamente en titulaciones de Grado del ámbito de la Educación y, desde un enfoque cuantitativo.

\section{Referencias bibliográficas}

Alexa (2020). wikipedia.org Competitive Analysis, Marketing Mix and Traffic. https://bit.ly/3h2zsuX

Alonso, M.I., \& García, J. (2013). Colaboración activa en Wikipedia como método de aprendizaje. RIED, 16(1), 13-26. https://doi.org/10.5944/ried.16.1.2056

Barnhisel, G., \& Rapchak, M. (2014). Wikipedia and the Wisdom of Crowds: A Student Project. Communications in Information Literacy, 8(1), 145-159. https://doi.org/10.15760/comminfolit.2014.8.1.158

Brailas, A., Koskinas, K., Dafermos, M., \& Alexias, G. (2015). Wikipedia in Education: Acculturation and learning in virtual communities. Learning, Culture and Social Interaction, 7, 59-70. http://dx.doi.org/10.1016/j.lcsi.2015.07.002

Brox, H. (2016). Troublesome tools: How can Wikipedia editing enhance student teachers' digital skills? Acta Didactica Norge, 10(2), 329-346. https://doi.org/10.5617/adno.2493

Brox, H. (2019). Why won't they take them on? A study on student teachers' first-time engagement with wiki technology. [Tesis doctoral, UiT The Arctic University of Norway]. Repositorio Institucional UiT. https://bit.ly/2VlRQ8I

Chen, H.L. (2010). The perspectives of higher education faculty on Wikipedia. The Electronic Library, 28(3), 361-373. https://doi.org/10.1108/02640471011051954 Christensen, T.B. (2015). Wikipedia as a Tool for 21st Century Teaching and Learning. International Journal for Digital Society, 6(2), 1042-1047. https://doi.org/10.20533/ijds.2040.2570.2015.0128

Cuquet, M., \& García-San Pedro, M.J. (2019). Percepciones y uso de la Wikipedia en alumnos de educación secundaria. Education in the knowledge society (EKS), 20. https://doi.org/10.14201/eks2019_20_a8

Dawe, L., \& Robinson, A. (2017). Wikipedia editing and information literacy: A case study. Information and Learning Science, 118(1/2), 5-16. https://doi.org/10.1108/ILS-09-2016-0067

Di Lauro, E., (2016). Employing Wikipedia for good not evil: Innovative approaches to collaborative writing assessment. Assessment \& Evaluation in Higher Education, 42(3), 478-491. http://dx.doi.org/10.1080/02602938.2015.1127322

Eijkman, H. (2010). Academics and Wikipedia: Reframing Web $2.0+$ as a disruptor of traditional academic power-knowledge arrangements. Campus-Wide Information Systems, 27(3), 173-185. https://doi.org/10.1108/10650741080000448 
Flanagin, A.J., \& Metzger, M.J. (2011). From Encycopaedia Britannica to Wikipedia. Generational differences in the perceived credibility of online encyclopedia information. Information, Communication \& Society, 14(3), 355-374. https://doi.org/10.1080/1369118X.2010.542823

Foglia, M. (2008). Wikipédia : Média de la connaissance démocratique? Quand le citoyen lambda devient encyclopédiste books. FYP

Forte, A., \& Bruckman, A. (2010). Writing, citing, and participatory media: Wikis as learning environments in the high school classroom. International Journal of Learning and Media, 1(4), 23-44. https://doi.org/10.1162/ijlm_a_00033

García, A.A. (2010). Wikis en tareas educativas: Aplicaciones de la Web 2.0 en Secundaria y Bachillerato [Tesis doctoral, Universidad de Salamanca] Repositorio Institucional US. https://doi.org/10.14201/gredos.83223

Garrison, J.C. (2018), Instructor and peer influence on college student use and perceptions of Wikipedia, The Electronic Library, 36(2), 237257. https://doi.org/10.1108/EL-02-2017-0034

Gilles, M. (2015). Wikipédia, une encyclopédie collaborative en quête de crédibilité: Le référencement en questions [Tesis doctoral, Universidad de Toulouse]. https://bit.ly/3cGZ2lO

Gourdain, P., O'Kelly, F., Roman-Arnat, B., Soulas, D., \& Von-Droste, T. (2007). La Révolution Wikipédia. Les Mille et Une Nuits

Hastings-Ruiz, D. (2015) Wikipedia and theories of knowledge in encyclopaedism. [Tesis doctoral, Universidad de Valladolid]. Repositorio Institucional UV. https://doi.org/10.35376/10324/16184

Head, A.J., \& Eisenberg, M.B. (2010a). How today's college students use Wikipedia for courserelated research. First Monday, 15(3). https://doi.org/10.5210/fm.v15i3.2830

Instituto Nacional de Estadística (16 de octubre de 2019). Encuesta sobre equipamiento y uso de tecnologías de información y comunicación en los hogares. https://bit.ly/30nVke6

Josefsson, P., Bälter, O., Bälter, K., \& Bonn, S. (2014). Attitude Changes When Using Wikipedia in Higher Education. En J. Viteli \& M. Leikomaa (Eds.), EdMedia: World Conference on
Educational Media and Technology (pp. 20242032). Association for the Advancement of Computing in Education (AACE).

Judd, T., \& Kennedy, G. (2011). Expediency-based practice? Medical students' reliance on Google and Wikipedia for biomedical inquiries. British Journal of Educational Technology, 42(2), 351-360. https://doi.org/10.1111/j.1467-8535.2009.01019.x

Kleimann, B., Özkilic, M., \& Göcks, M. (2008). Studieren im Web 2.0. Studienbezogene Web- und E-Learning-Dienste. HISBUSKurzinformation, 21. Hanover. https://bit.ly/2MJ95w9

Lim, S. (2013). Does formal authority still matter in the age of wisdom of crowds?: Perceived credibility, peer and professor endorsement in relation to college students. Wikipedia use for academic purposes. American Society for Information Science and Technology, 50(1), $1-4$. https://doi.org/10.1002/meet.14505001118

Lladós, J., Aibar, E., Lerga, M., Meseguer-Artola, A., \& Minguillón, J. (2013). An Empirical Study on Faculty Perceptions and Teaching Practices of Wikipedia. In M. Augier \& M. Ciussi (Eds.), 12th European Conference on e-Learning. Academic Conferences and Publishing International Limited (pp. 258-265).

https://bit.ly/3eX3RZH

Los cinco pilares (3 de mayo de 2020). In Wikipedia. https://bit.ly/3dCeWPk

Margaryan, A., Littejohn, A., \& Vojt, G. (2011). Are digital natives a myth or reality? University students use of digital technologies. Computers \& Education, 56(2), 429-440. https://doi.org/10.1016/j.compedu.2010.09.004

Meishar-Tal, H. (2015). Teachers' use of Wikipedia with their Students. Australian Journal of Teacher Education, 40(12). https://doi.org/10.14221/ajte.2015v40n12.9

Meseguer-Artola, A. (2014). Aprendiendo mediante la comparación con Wikipedia: Su importancia en el aprendizaje de los estudiantes. RUSC. Universities and Knowledge Society Journal, 11(2), 57-69. http://dx.doi.org/10.7238/rusc.v11i2.2042

Meseguer-Artola, A., Rodríguez-Ardura, I., Ammetller, G., \& Rimbau-Gilabert, E. (2019). Learning 
with Wikipedia in Higher Education: Academic Performance and Students' Quality Perception. In A. Visvizi \& M. Lytras (Eds.), Research \& Innovation Forum 2019. RIIFORUM 2019. Springer Proceedings in Complexity. Springer, Cham.

https://doi.org/10.1007/978-3-030-30809-4_12

Obregón, A., \& González, N. (2016). Validación de un cuestionario piloto para evaluar el conocimiento de la Wikipedia en estudiantes de Educación Superior. Revista del Congrés Internacional de Docència Universitària $i$ Innovació (CIDUI). https://bit.ly/307DRqh

Park, T.K. (2011). The visibility of Wikipedia in scholarly publications. First Monday. Peer Reviewed Journal on the Internet, 16(8). https://doi.org/10.5210/fm.v16i8.3492

Petrucco, C. (2019). Wikipedia as complementary formative assessment method in University Courses. In J. Theo Bastiaens (Ed.), Actas del EdMedia + Innovate Learning (pp. 335338(. Association for the Advancement of Computing in Education (AACE). https://bit.ly/2AM4BSH

Pozzali, A., \& Ferri, P. (2010). Old and new media in the everyday life in University students in Italy. Revista de Informatica Sociala, 14, 9-20. https://bit.ly/2UeXybR

Sahut, G., Jeunier, B., Mothe, J., \& Tricot, A. (2015). Qu'apprennent les jeunes usagers á propos de Wikipédia? In L. Barbe, L. Merzeau \& V. Schafer, Wikipédia objet scientifique non identifié. Presses universitaires de Paris Ouest (pp. 149-161). https://bit.ly/373ewPz

Sánchez, L. (2014). Utilización de Wikipedia como recurso docente en la enseñanza superior [Tesis doctoral, Universidad de Salamanca]. Repositorio institucional US. https://doi.org/10.14201/gredos.125932

Selwyn, N., \& Gorard, S. (2016). Students' use of Wikipedia as an academic resource - Patterns of use and perceptions of usefulness. The Internet and Higher Education, 28, 28-34. http://dx.doi.org/10.1016/j.iheduc.2015.08.004

Shen, X.L, Cheung, C.M.K., \& Lee, M.K.O. (2013). What leads students to adopt information from Wikipedia? An empirical investigation into the role of trust and information usefulness. British Journal of Educational Technology, 44(3), 502-517. https://doi.org/10.1111/j.1467-8535.2012.01335.x Soler-Adillon, J., Pavlovic, D., \& Freixa, P. (2018). Wikipedia en la Universidad: Cambios en la percepción de valor con la creación de contenidos. Comunicar, 54, 39-48. https://doi.org/10.3916/C54-2018-04

Sormunen, E., \& Lehtiö, L. (2011). Authoring Wikipedia articles as an information literacy assignment: Copy-pasting or expressing new understanding in one's own words? Information Research, 16. https://bit.ly/2MsLxLM

Valverde-Crespo, D., \& González-Sánchez, J. (2016). Búsqueda y selección de información en recursos digitales: Percepciones de alumnos de Física y Química de Educación Secundaria Obligatoria y Bachillerato sobre Wikipedia. Revista Eureka sobre enseñanza y divulgación de las ciencias, 13(1), 67-83.

https://doi.org/10.25267/rev_eureka_ensen_divulg_cienc.2016.v13.i1.06

Valverde-Crespo, D., González-Sánchez, J., \& ProBueno, A. (2019). Wikipedia en la Universidad: ¿Cómo la utilizan los estudiantes de $1^{\circ}$ curso de Grado de titulaciones del área de Ciencias Experimentales? Un estudio sobre sus percepciones. Revista Eureka sobre enseñanza y divulgación de las ciencias, 16(3), 1-18.

https://doi.org/10.25267/Rev_Eureka_ensen_divulg_cienc.2019.v16.i3.3101

Wannemacher, K., \& Schulenburg, F. (2010). Wikipedia in Academic Studies: Corrupting or Improving the Quality of Teaching and Learning? In M. Ebner \& M. Schiefner (Eds.), Looking Toward the Future of Technology-Enhanced Education: Ubiquitous Learning and the Digital Native (pp. 295-310). IGI Global. https://doi.org/10.4018/978-1-61520-678-0.ch017

Zou, D., Xie, H., Wang, F.L., \& Kwan, R. (2020) Flipped learning with Wikipedia in higher education, Studies in Higher Education, 45(5), 1026-1045.

https://doi.org/10.1080/03075079.2020.1750195 